\title{
Optical fiber vacuum sensor based on etched SMS structure and PDMS coating
}

\author{
Joaquin Ascorbe ${ }^{1}$, Omar Fuentes ${ }^{2,3}$, Francisco J. Arregui ${ }^{2,3}$, Member, IEEE, Ignacio R. Matias ${ }^{2,3}$ Senior Member, \\ IEEE, and Jesus M. Corres ${ }^{2,3}$, Member, IEEE \\ ${ }^{1}$ Nadetech Innovations S.L. SPAIN \\ ${ }^{2}$ Department of Electrical, Electronic and Communication Engineering, Public University of Navarra, SPAIN \\ ${ }^{3}$ Institute of Smart Cities, Public University of Navarra, SPAIN
}

\begin{abstract}
In this work, an optical fiber vacuum sensor based on a single-mode multimode single-mode (SMS) structure coated with polydimethylsiloxane (PDMS) is studied. The SMS structure generates an interferometric pattern based on multimode interference. The structure is dip-coated with a layer of PDMS, whose optical properties change when it is subjected to varying vacuum pressure. Different strategies are applied in an attempt to improve the final performance of the sensor, such as decreasing the diameter of the fiber and modifying the properties of the coating by modifying the proportion of solvent. Decreasing the diameter of the optical fiber and using toluene as a solvent are both proved to be successful strategies for increasing the sensitivity of the sensor. The devices are studied in the $1 \times 10^{-3}-10$ mbar range with a maximum wavelength shift of $12 \mathrm{~nm}$, leading to a maximum sensitivity of $35 \mathrm{~nm} / \mathrm{mbar}$. The simplicity of the fabrication process, which can be applied to more sensitive structures, suggests that PDMS may be a good choice for the development of optical fiber vacuum sensors.
\end{abstract}

Keywords - optical fiber; modal interferometer; vacuum sensor; polydimethylsiloxane; PDMS;

\section{INTRODUCTION}

A large number of industrial, healthcare and scientific applications [1] require control over vacuum conditions in order to operate correctly. For vacuum measurement, one of the most widely used technologies is a Pirani gauge, which is based on thermal conductivity [2], and allows the user to measure pressures of up to $1 \times 10^{-4} \mathrm{mbar}$. Lower pressures require special gauges, such as cold or hot cathode gauges, which allow for the measurement of pressures of up to $1 \times 10^{-9}$ and $1 \times 10^{-12}$ mbar, respectively [3].

However, these types of sensor may have limited usage in harsh environments, where the advantages of optical fiber sensors are clear, and particularly their immunity to electromagnetic fields and their size. Within the variety of optical fiber structures utilized for vacuum pressure sensing, different configurations and working principles can be found. Fabry-Pèrot interferometers are constructed using a vacuumsealed cavity whose length varies as a function of the pressure [4]. The diaphragm is created by following a process that includes several steps, such as photo-resist patterning and reactive ion etching (RIE). An easier setup was used by Wang et al., who spliced a hollow core to a single-mode fiber (SMF) and then added a diaphragm, also made with a fiber segment [5]. This scheme was used to measure positive pressures; it did not

This work was supported by the Spanish Agencia Estatal de Investigación (AEI), Fondo Europeo de Desarrollo Regional (FEDER) (TEC2016-79367-C22-R) and a Torres-Quevedo grant from the Science and Innovation Ministry (PTQ2018-010221). have a high sensitivity, and only changes in power could be distinguished, although it was a robust structure. Another optical structure that is appropriate for vacuum sensing is a fiber Bragg grating (FBG) [6], which has a high sensitivity to strain and temperature. Here, the working principle is similar to that of the Pirani gauge, as it consists of heating the metal-coated FBG by directing a laser at it [6] and measuring the difference in the transferred heat.

Unlike previous approaches, in this work we investigate the performance of polydimethylsiloxane (PDMS) as an optical transducer for vacuum measurements. The use of this type of silicone as the sensitive component in the device has some advantages, such as the independence of the measurements of the power of the light source, which can be determined for those sensors based on the transferred heat.

In order to obtain an optical signal of sufficient strength when the pressure changes, we use an SMS structure in this paper. It consists of two single-mode fibers (SMFs) that are spliced to the ends of a section of a multimode coreless fiber (MMF). This structure is simple to fabricate, and gives sensors with adequate sensitivity [7]-[11]. The SMS structure generates a modal interferometer that is sensitive to several parameters such as temperature [11], strain [9], [12]-[14] and refractive index [8]. When used as a refractometer, a value of 1200 $\mathrm{nm} / \mathrm{RIU}$ has been reported for a range of refractive indices from 1.321 to 1.382 [8]. In addition, it has been previously demonstrated that reducing the diameter of the fiber by chemical etching leads to an increase in the sensitivity [10] due to a stronger interaction between the evanescent field and the surrounding medium [15]. This improvement in the sensitivity depends exclusively on the diameter rather than on the length of the MMF segment [7], [10]. Although they are not discussed in this work, there are other possibilities for improving the interference pattern of this kind of interferometer, such as the use of few-mode fibers [16] rather than an MMF.

The PDMS coating is cured at atmospheric pressure. Due to its elastic properties, vacuum pressure causes the air trapped in the PDMS layer to expand it, thus modifying its refractive index, and to a lesser extent inducing strain in the fiber. Variations in the proportions of the base and curing agent can provide different sensitivities, due to changes in the degree of crosslinking of the polymer. Different PDMS co-polymers can also provide different elastic properties. Significant changes in the Young modulus (E) can be observed, which are caused by the 
different percentages of short chains in the polymer network [17]. The deformation capability of the coating under pressure conditions, and hence the performance of the sensor, can be affected by both the drying temperature and the addition of a solvent such as toluene to the PDMS after the mixing stage [18].

\section{FABRICATION PROCESS}

The length and diameter of the MMF segment are critical parameters in the design of the optical sensor, specifically in order to obtain a sinusoidal spectrum with sufficient amplitude to allow wavelength measurements and a fast Fourier transform analysis to be performed. The first step in fabricating this device is to cut a piece of MMF (POFC, Inc.) to the desired length at an angle of $90^{\circ}$ on both sides. This segment is then spliced to two standard SMF fiber pigtails (Telnet, Inc.). The outer diameter of all fibers is $125 \mu \mathrm{m}$. The structure is then etched using hydrofluoric acid (40\% HF) until the desired diameter of the fiber is obtained.

The first length tested was $15 \mathrm{~mm}$, as this gave a good interference pattern when the diameter was reduced to $30 \mu \mathrm{m}$ [8]. If the diameter was reduced below this value, the amplitude of the sinusoidal signal rapidly started to decrease. For this reason, in order to reduce the diameter to $20 \mu \mathrm{m}$, the length needed to be shortened to $6 \mathrm{~mm}$. This was verified both theoretically and experimentally. The etched SMS was washed with deionized water to remove the remaining acid. The etching process was monitored in order to stop it when the transmitted optical spectrum achieved the desired characteristics of a quasisinusoidal spectrum, since this behavior facilitates the phaseshift analysis.
Finally, the resulting structure was attached to a U-holder and the PDMS layer was added. Two different brands of PDMS were used, each with a different tensile strength: Sylgard 184 [19] and RTV-615. The PDMS was prepared by mixing the elastomer base with the curing agent in a ratio of 10:1. Following this, the mixture was dissolved in toluene $(2: 1)$ to obtain a solution with lower viscosity, and hence to provide greater control at a later stage over the thickness and shape of the coating made by dip-coating. The dip-coating process was carried out manually in this first approach. Both the immersion and withdrawal processes were performed in the slowest possible way, and one dip was considered sufficient for each sensor fabricated. Due to the viscosity of the material, there was high level of surface tension, and dip-coating was therefore a delicate process. The withdrawal speed and the time between the preparation and application of the material had a strong influence on the morphological properties of the coating. Immediately after coating, the device was heated to $100^{\circ} \mathrm{C}$ to dry the layer at atmospheric pressure. The final thicknesses obtained were between 5 and $16 \mu \mathrm{m}$.

The experimental apparatus used for the characterization of this device (shown in Fig. 1) was a typical transmission set-up, consisting of a light source (Pyroistech.SL FJORD-X2-13301550) and an optical spectrum analyzer (HP 86142A). The sensor was placed inside a vacuum chamber; this had an optical feedthrough in which a vacuum was achieved using a rotary vacuum pump and a turbo-molecular pump. The vacuum pressure was acquired using a data-logger (Agilent 34972A) and a vacuum pressure sensor (Pfeiffer PKR251), which consists of calibrated Pirani and cold-cathode gauges.

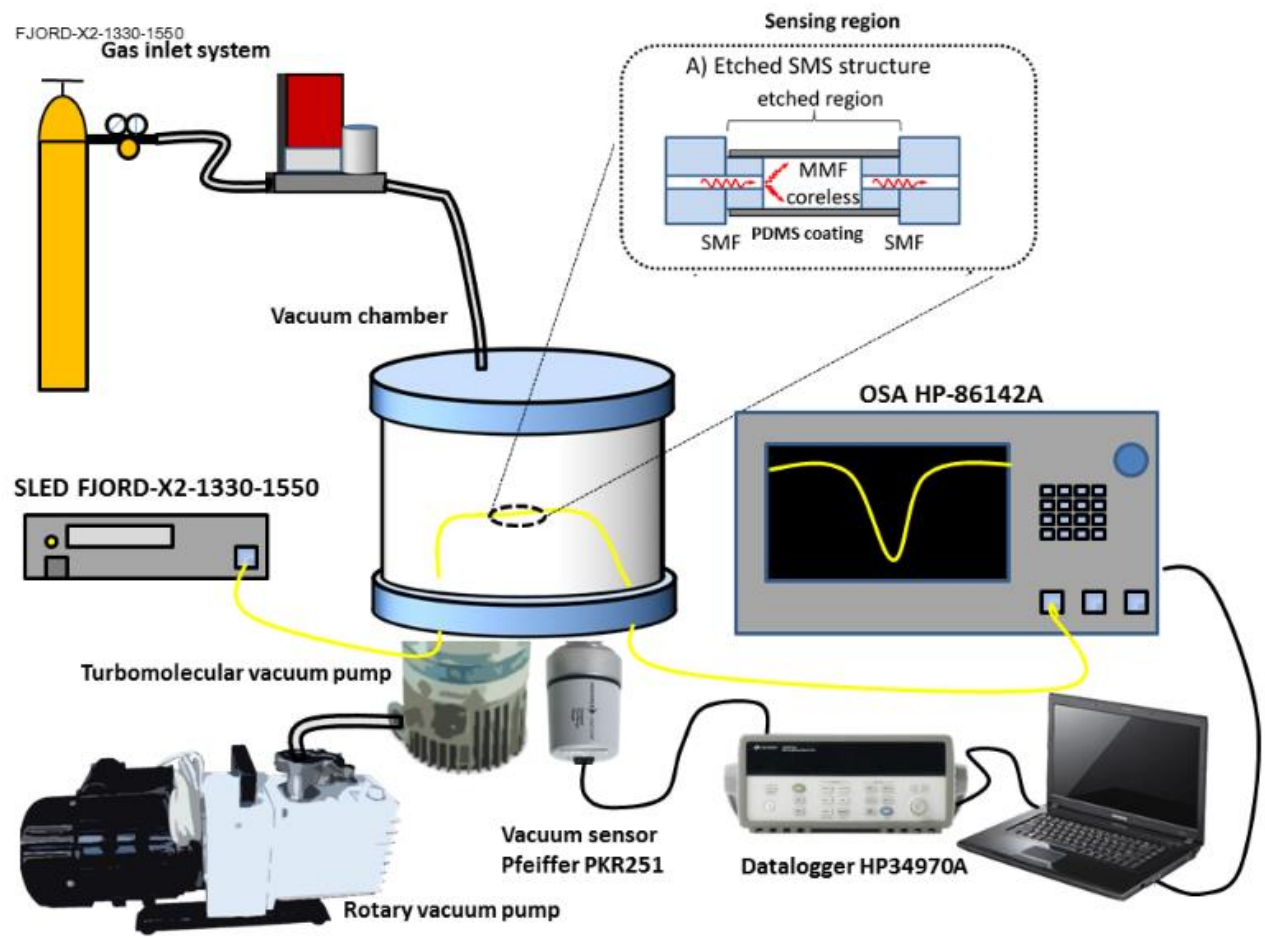

Fig. 1. Experimental set-up used for the characterization of the etched SMS vacuum sensors 


\section{EXPERIMENTAL RESULTS}

\section{A. Fabrication process}

As is well known, SMS interferometers can be tuned to work in two modes of operation. One of these is the self-image effect [13], which produces a maximum in the transmission spectrum that can be distinguished from other maxima by the presence of fractional constructive interference. The operating mechanism of this modal interferometer is based on the excitation of several eigenmodes of the MMF, which leads to interference among several modes during the propagation along the MMF section. Finally, the light is coupled back into the output SMF.

The second mode of operation of the SMS structure permits it to be used as both a wavelength and a phase-sensitive device. Due to the reduction in the diameter of the MMF section, the evanescent field penetrates further into the surrounding medium, thus increasing the sensitivity [10].

In fact, according to [8], the diameter is inversely proportional to the sensitivity to the refractive index. Consequently, a reduction from 125 to $30 \mu \mathrm{m}$ is expected to lead to a 4.16 fold increase, and a reduction from 125 to $20 \mu \mathrm{m}$ to a 6.25 fold increase, assuming that the modification of the PDMS properties is due only to changes in the refractive index.

Three different devices were fabricated and tested. The difference between devices D1 and D2 was due to the presence or absence of toluene in the PDMS solution. Device D3 differed from D1 and D2 both in terms of the dimensions of the fiber and in the brand of PDMS used.

Fig. 2 shows the evolution of the transmitted optical spectrum while the etching process was taking place. Fig. 2 (a) corresponds to the MMF section that was $15 \mathrm{~mm}$ in length, and Fig. 2 (b) to the $6 \mathrm{~mm}$ section. Here, it is important to remark that the different lengths were determined in such a way that the diameter could be reduced while still giving a good interference pattern. More detailed information about how the different parameters can affect the SMS in terms of the transmitted optical spectrum and sensitivity can be found in [7], [21], [22].

From Fig. 2, it is possible to observe how the spectrum evolves while the etching process is taking place: the attenuation bands undergo a blueshift, and more bands start to appear from greater wavelengths, which are also subject to a blueshift. These attenuation bands begin to separate, and finally start to lose amplitude, forming a quasi-sinusoidal shape.
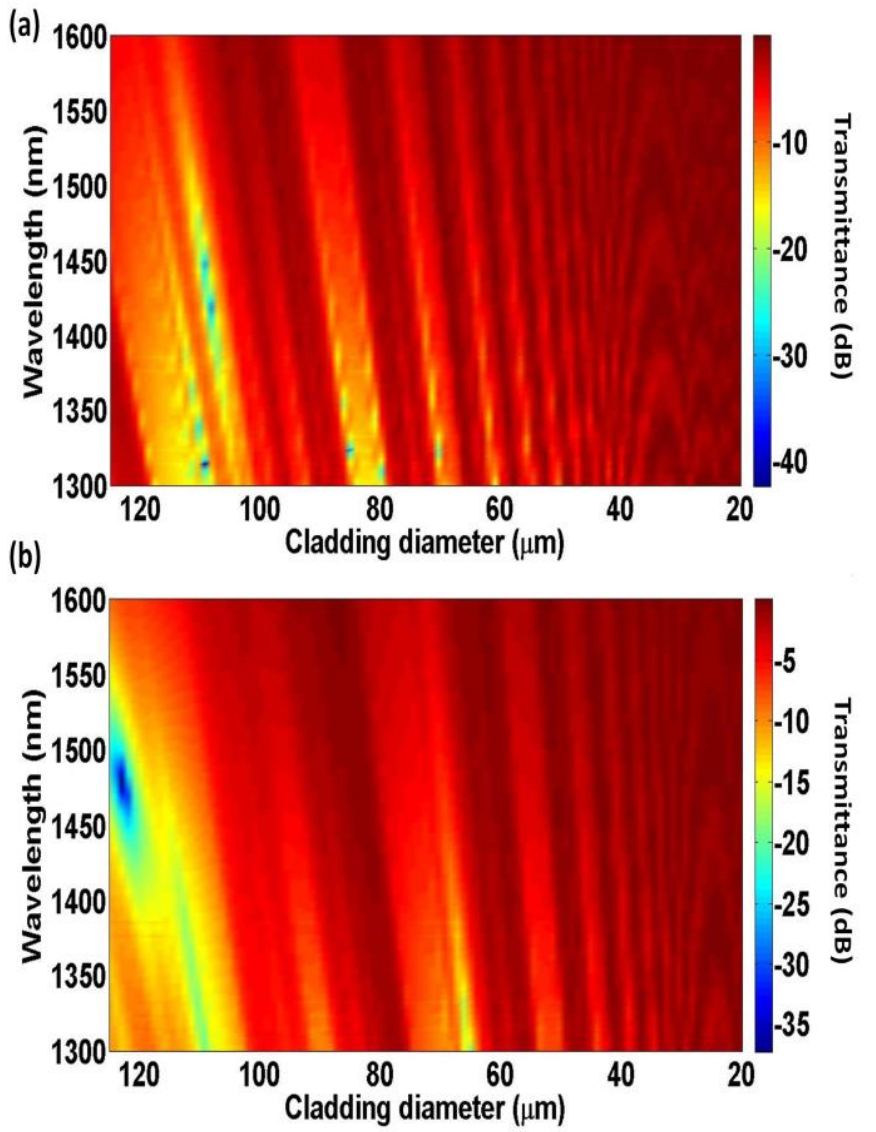

Fig. 2. Evolution of the transmitted optical spectrum at different diameters of the fiber for (a) the $15 \mathrm{~mm}$ devices (D1, D2) and (b) the $6 \mathrm{~mm}$ device (D3)

From a comparison of sensors D1 and D3, it can be observed that although the evolution of the spectrum is similar, it takes a shorter time for the longer device, which agrees with the requirement to use shorter lengths to give smaller diameters. On the other hand, longer devices provide better interference in terms of the spectral width and free spectral range, as can be observed from Fig. 3. However, since the sensitivity is only determined by the overall diameter, a device of smaller diameter should provide better results. When the etching process was finished, the PDMS layer was added by dip-coating. Due to the addition of a high refractive index layer (i.e. the PDMS coating), the spectrum shifts, as can be observed from Fig. 3. The estimated thickness of the coating, which is greater than $5 \mu \mathrm{m}$, means that it acts as an infinitum medium, because the thickness is greater than the penetration depth of the evanescent field. Table I summarizes the experimental details used for the three devices used here, ordered based on their pressure sensitivity. 

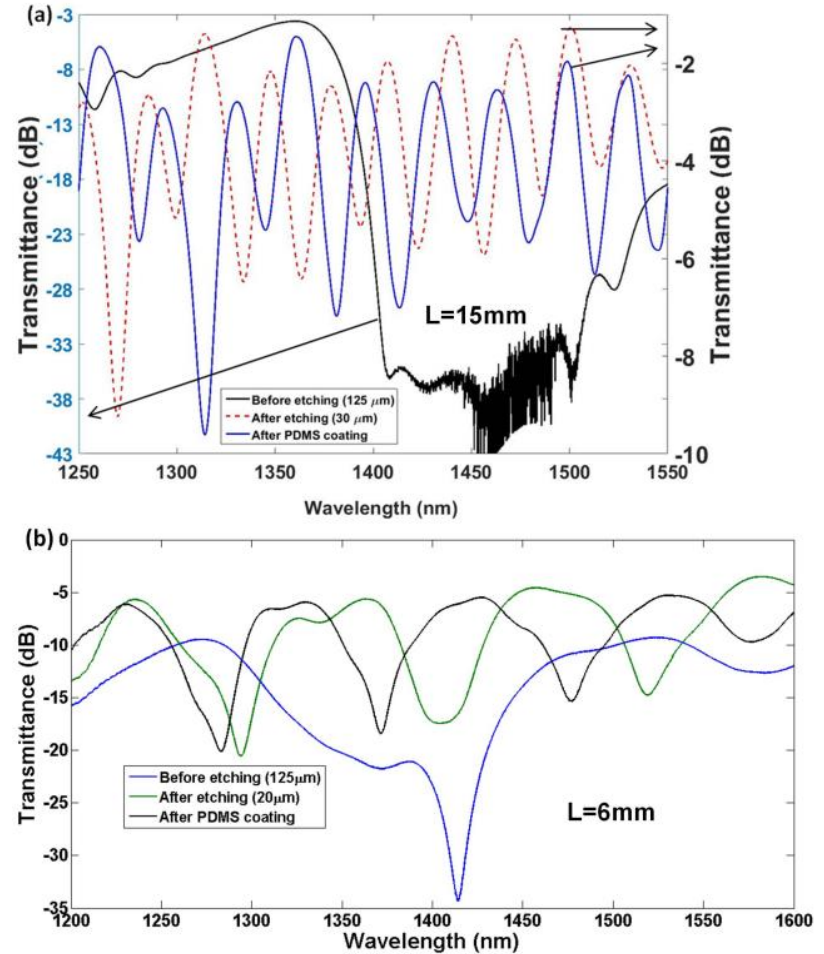

Fig. 3. Optical spectrum transmitted at each of the different steps in the fabrication process of the devices with (a) $15 \mathrm{~mm}$ length, $30 \mu \mathrm{m}$ diameter (D1, D2) and (b) $6 \mathrm{~mm}$ length, $20 \mu \mathrm{m}$ diameter (D3)

As can be observed from Fig. 3 (a), the final spectrum of the device of length $15 \mathrm{~mm}$ shows a maximum peak of $6.5 \mathrm{~dB}$ in the amplitude and a full width at half minimum (FWHM) of $10 \mathrm{~nm}$, and consequently a quality factor $(\mathrm{Q})$ of 0.65 , whereas the device of length $6 \mathrm{~mm}$ has an amplitude of $14 \mathrm{~dB}$ and a FWHM of 13 $\mathrm{nm}$, giving a better $\mathrm{Q}$ factor of 1 . In addition, the free spectral range (FSR) of the shorter device $(90 \mathrm{~nm})$ is greater than that of the longer device $(30 \mathrm{~nm})$, which facilitates measurement.

Table I. Parameters of the three devices tested

\begin{tabular}{|c|c|c|c|c|c|c|c|}
\hline & Supplier & $\begin{array}{l}\text { Base/ } \\
\text { curing }\end{array}$ & $\begin{array}{c}\text { Toluene/ } \\
\text { PDMS }\end{array}$ & $\begin{array}{c}\Phi \\
\mu \mathrm{m}\end{array}$ & $\underset{\mathbf{m m}}{\mathrm{L}}$ & $\underset{\mathrm{nm} / \mathrm{mbar}}{\mathrm{S}}$ & $\begin{array}{l}\text { Pressure } \\
\text { range mbar }\end{array}$ \\
\hline D1 & Sylgard & $10: 1$ & $0: 1$ & 30 & 15 & $<0.05$ & - \\
\hline D2 & Sylgard & $10: 1$ & $2: 1$ & 30 & 15 & 11.4 & $0.63 \rightarrow 0.079$ \\
\hline D3 & RTV615 & 10:1 & $2: 1$ & 20 & 6 & 35 & $0.63 \rightarrow 0.016$ \\
\hline
\end{tabular}

Due to the viscosity of the PDMS [12], a group of almost periodic elevations can be observed (see Fig. 4). The uniformity of the layer can be increased by using toluene as a solvent, although its influence on the sensitivity at higher proportions needs to be analyzed further.

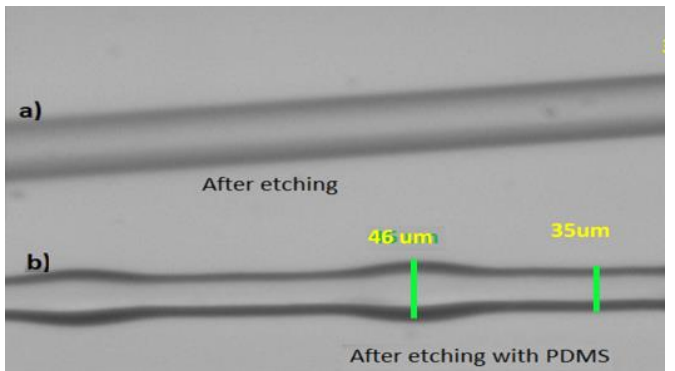

Fig. 4. Image of the SMS structure (a) after chemical etching (diameter $30 \mu \mathrm{m}$ ), and (b) after chemical etching and with PDMS coating

\section{B. Pressure measurement}

The procedure for characterizing the pressure sensors is as follows. The rotary vacuum pump is started, and it is then necessary to wait until the pressure drops to $3 \times 10^{-1}$ mbar; the turbo-molecular pump is then turned on and operated until the vacuum pressure reaches almost $1 \times 10^{-3}$ mbar. To return to atmospheric pressure, the vacuum pumps are switched off in reverse order, and a gas flow is used to finally recover the initial state.

For faster optical measurements, the span of the optical spectrum was reduced, and the measurements were performed at around $1310 \mathrm{~nm}$.

\section{1) Device DI}

Device D1 was fabricated without using toluene as a solvent. The dynamic response plotted in Fig. 5 shows the low sensitivity of this sensor. The left axis corresponds to the wavelength of a peak, whereas the right axis represents the decimal logarithm of the pressure in mbar. There is a wavelength shift of only $0.6 \mathrm{~nm}$ when the device pressure is changed between vacuum and atmospheric pressure. As can be seen from Fig. 6, this device is almost immune to changes in pressure.

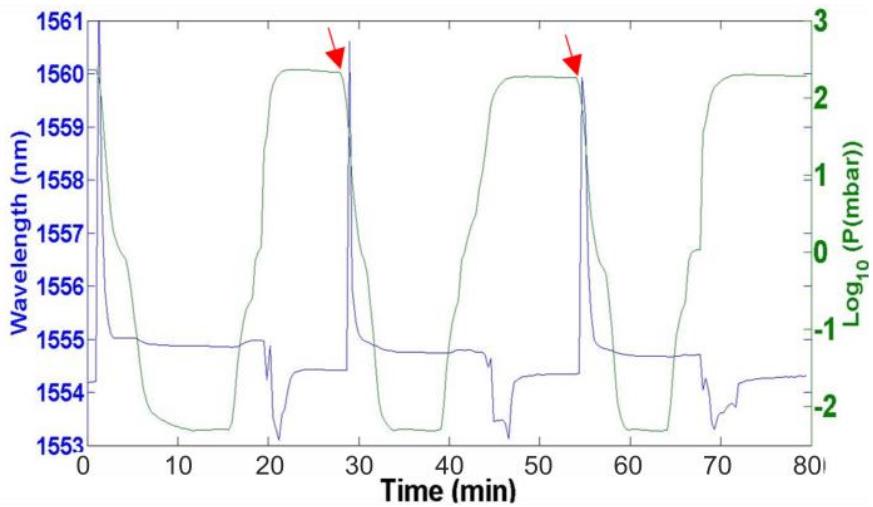

Fig. 5. Dynamic response of the sensor (D1). Red arrows indicate the starting times of the rotary pump 


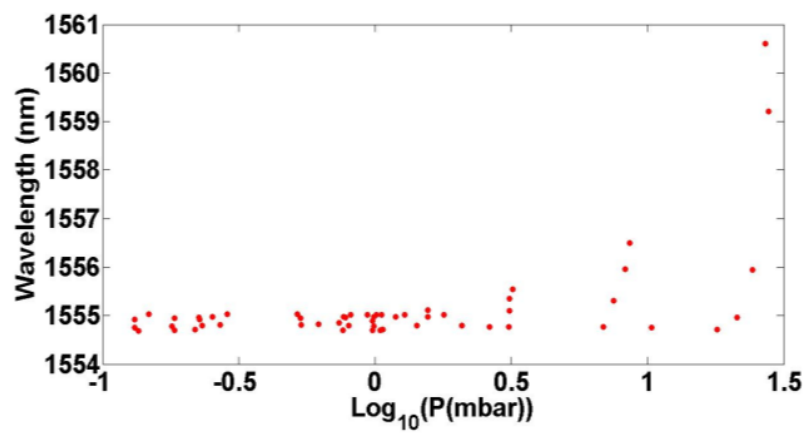

Fig. 6. Characterization of the sensor D1

From a comparison with the remaining devices, we can conclude that toluene must play some role in the elastic behavior of the material; this may be during the coating process, as it evaporates quickly, providing a less dense coating or reducing its stiffness.

\section{2) Device D2}

The next device, D2, was similar to the previous one except that toluene was used to dissolve the elastomer/base mix. Fig. 7 shows the dynamic response of the optical fiber vacuum sensor as the pressure changes. The changes in the wavelength and phase of the interferometer follow the changes in the pressure measured by the commercial sensor. As discussed above, this effect is achieved due to the use of toluene as a solvent. The response time seems to be lower than that of the commercial sensor.

Since modifying the pressure in a vacuum chamber is not an immediate process, and as the commercial sensor has its own response time, it was not possible to calculate the exact response time of the proposed device, although it was possible to carry out a comparison of the response times of both sensors using their respective slopes and their dynamic ranges. It can be observed from Fig. 7 that the optical fiber sensor has a slope of $0.04 \mathrm{~nm} / \mathrm{s}$ and an increment of $3.1 \mathrm{~nm}$, whereas Pirani gauge has a slope of $0.0183 \mathrm{mbar} / \mathrm{s}$ and an increment of $2.2477 \mathrm{mbar}$. Previous data lead to a relative speed of $0.0129 \mathrm{~s}^{-1}$ and $0.0082 \mathrm{~s}^{-}$ ${ }^{1}$ for the optical fiber sensor and the Pirani gauge, respectively, which means that the optical fiber sensor is a 50\% faster than the Pirani gauge.

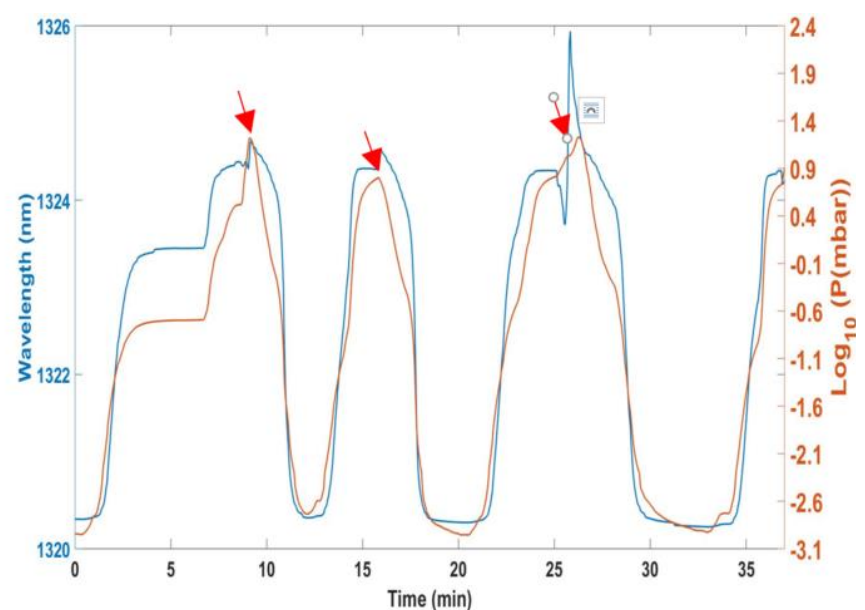

Fig. 7. Dynamic response of the optical fiber vacuum sensor (D2) for pressures ranging from 16 to $1.1 \times 10^{-3}$ mbar. Red arrows indicate the starting times of the rotary pump

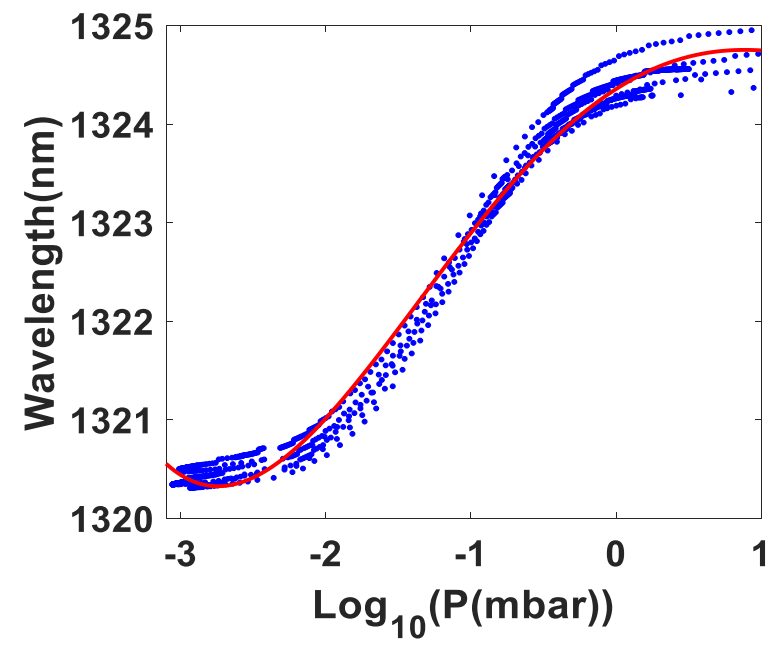

Fig. 8. Wavelength shift of an attenuation band as a function of the vacuum pressure of D2. (the red line is the $4^{\text {th }}$ degree polynomial fitting)

From an analysis of Fig. 7, it can be observed that this sensor has good reversibility as well as high stability. It can also be seen that there is a peak when the rotary pump is started, which is also present for the commercial sensor. Finally, it is clear that the range of this sensor is lower than that of the commercial sensor. All of these aspects can be seen more clearly in Fig. 8, which shows the wavelength shift as a function of the vacuum pressure.

From Fig. 8, it can also be observed that the resonance wavelength is blueshifted as the pressure decreases. Greater sensitivity is obtained in the 0.01 to 0.3 mbar range, where the device shows linear behavior with a sensitivity of 11.43 $\mathrm{nm} / \mathrm{mbar}$ or $2.1744 \mathrm{~nm} / \log (\mathrm{mbar})$. At greater or smaller pressures, the PDMS layer does not suffer the same deformation and changes in refractive index, and the sensitivity is therefore reduced.

\section{3) Device D3}

In the final device, D3, the diameter of the MMF segment was reduced to $20 \mu \mathrm{m}$, which is known to be a factor that increases the sensitivity. The dynamic response of device D3 is 
shown in Fig. 9. As for the previous device (D2), this sensor shows good reversibility.

In a similar way to that described above, the response time of device D3 was calculated and compared with device D2. Sensor D3 is shown to have a response that is twice as fast as D2, which may be caused by the differences in the elastic behavior of the material.

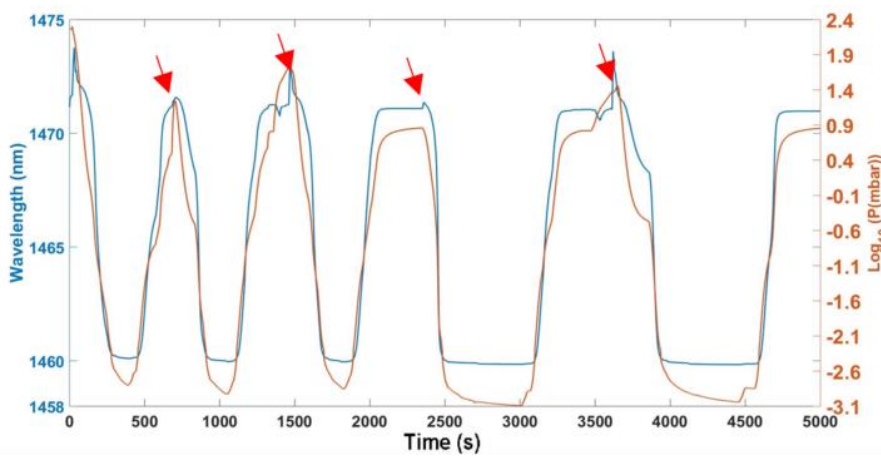

Fig. 9. Dynamic plot of the response of D3, when subjected to pressure changes. Red arrows indicate the starting times of the rotary pump

Compared with the graph in Fig. 7, it can be seen that the dynamic range of the sensor D3 is greater than that of D2. The wavelength shift reaches values of $12 \mathrm{~nm}$, giving sensitivities of $35 \mathrm{~nm} / \mathrm{mbar}$ or $5.5723 \mathrm{~nm} / \log (\mathrm{mbar})$. These values can be extracted from the maximum slope of the curve in Fig. 10, which can be used to characterize this device.

In the same way as for D2, the pressure range is limited, although higher sensitivity is seen at slightly greater pressures; this is probably due to the differences in the material.

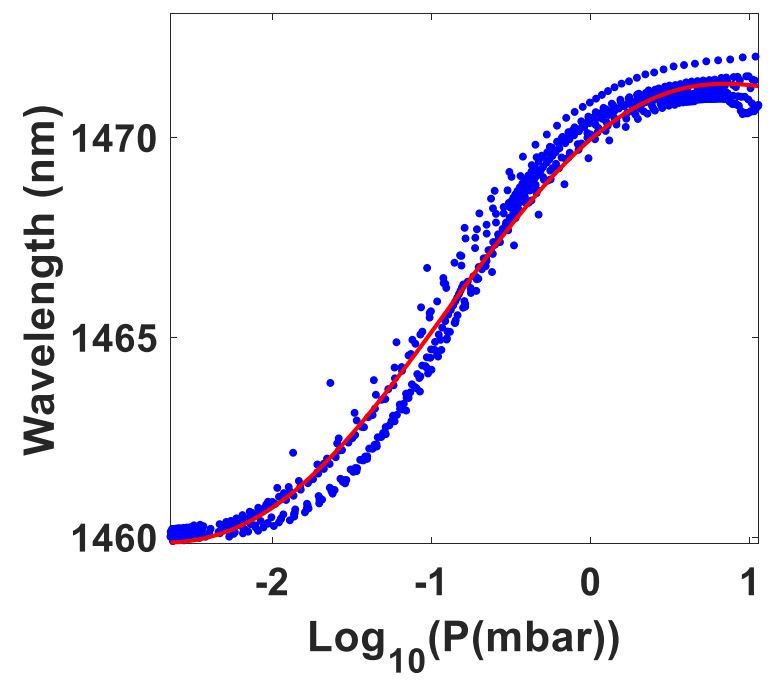

Fig. 10. Wavelength shift of an attenuation band as a function of the vacuum pressure for D3 (the red line is the $4^{\text {th }}$ degree polynomial fitting)

Fig. 11 shows a comparative graph on which the characterization results for devices D2 and D3 are plotted together. As can be seen, the sensitivity of device D3 (blue squares) is three times greater than that of D2 (red dots), which implies that there are other factors besides the diameter reduction that act to increase the sensitivity of this sensor. These are likely to include the mechanical properties of the material.

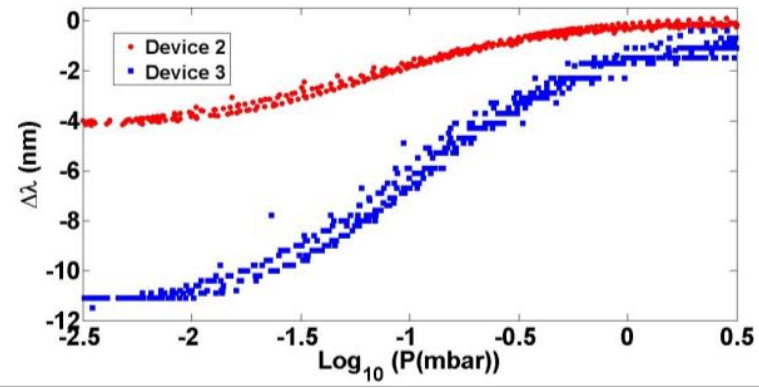

Fig. 11. Comparative graph of the characterization results for D2 (red dots) and D3 (blue squares)

\section{NUMERICAL SIMULATION}

In this section, a theoretical analysis will be performed for two of the devices presented in this work. The material used in both the simulations and the experiments was PDMS, which was deposited by means of the dip-coating technique. This material has small differences in the refractive index depending on the supplier. The refractive index of RTV615 was 1.406 , while for Sylgard 184, a value of 1.403 was found. It is assumed here that the external medium was entirely composed of PDMS, as its thickness was greater than the penetration depth of the evanescent field. FIMMWAVE® software was used for the numerical analysis of the structure. The RI of the optical fiber cladding, made of fused silica, was estimated using the Sellmeier equation. In the simulations, the RI of the optical fiber core was obtained, according to the specifications provided by Corning ${ }^{\circledR}$, by increasing the RI of the cladding by $0.36 \%$. The theoretical and experimental results were compared in order to extract relevant conclusions about the performance of the sensor. Two different devices were analyzed: a device with an MMF section of length $15 \mathrm{~mm}$ and diameter $30 \mu \mathrm{m}$, and another device with an MMF section of length $6 \mathrm{~mm}$ and diameter 20 $\mu \mathrm{m}$.

First, we compared the theoretical transmitted optical spectra obtained before and after the etching process of the fiber and the spectrum of the coated etched fiber, and these are shown in Fig. 12. In spite of the difficulty in ensuring the length of the MMF section, there was good agreement between both the theoretical and the experimental results with regard to the fabrication process (see Fig. 3). This can therefore be taken as a validation of the proposed model.

It can be observed that there are differences in the obtained spectra, as discussed in the experimental section: the device with a longer MMF section and larger diameter has a spectrum with more defined peaks and a better Q-factor and FSR, which are beneficial in terms of measurement, while the smaller device has a quasi-sinusoidal spectrum. Another theoretical simulation was carried out in order to study the sensitivity of the two different devices under analysis. For this purpose, and taking into account the fact that the coating conforms to the external medium, the refractive index of the PDMS layer was modified to give values of between 1.373 and 1.403, its nominal value. 


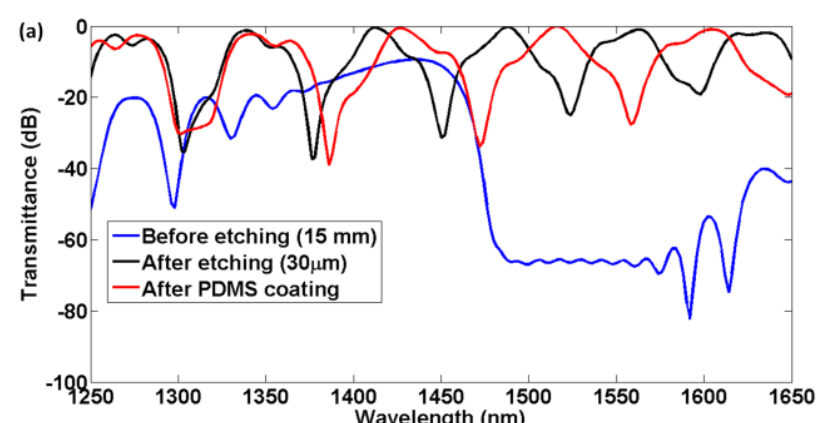

(b)

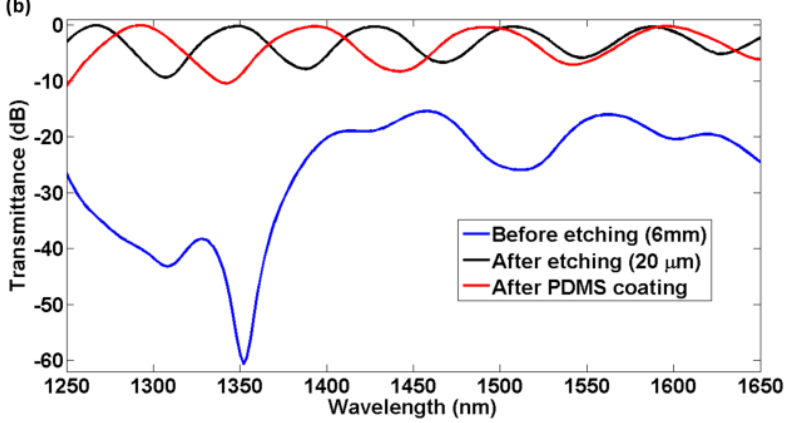

Fig. 12. Theoretical transmitted spectrum for SMS structures before etching, after etching and after coating with PDMS, for devices with (a) length $15 \mathrm{~mm}$ and diameter $30 \mu \mathrm{m}$, and (b) length $6 \mathrm{~mm}$ and diameter 20 $\mu \mathrm{m}$

As can be observed from Fig. 12 (a), the simulated spectrum for the device with length $15 \mathrm{~mm}$ shows a maximum peak of 30 $\mathrm{dB}$ of amplitude (as compared with $10 \mathrm{~dB}$ in the experimental case), a FWHM of $2 \mathrm{~nm}$ (10 nm for the real case) and a FSR of $86 \mathrm{~nm}$ (30 nm experimentally). Important differences can be found with regard to the experimental data, specifically in the amplitude and the FSR values. These differences can be attributed to the refractive index of the simulated PDMS, which may have a small extinction coefficient that has not been taken into account. It is also important to remark that in the simulated results, the thickness of the PDMS layer was assumed to be uniform, although it was known that this was not the case.

Fig. 12 (b) shows better agreement between the experimental and simulated results. Here, the amplitude was $10 \mathrm{~dB}$ versus 14 $\mathrm{dB}$ obtained experimentally, the FWHM was $28 \mathrm{~nm}$ (compared with $13 \mathrm{~nm}$ ) and the FSR was $100 \mathrm{~nm}$ (whereas experimentally it was $90 \mathrm{~nm}$ ). This better agreement between the experimental and simulated data can be attributed to the more homogeneous coating due to the shorter length of the device and to the differences in the properties of the material, such as its viscosity (3500 cPs for Sylgard; $4000 \mathrm{cPs}$ for RTV615) and tensile strength (71 versus $65 \mathrm{~kg} / \mathrm{cm}^{2}$ ).

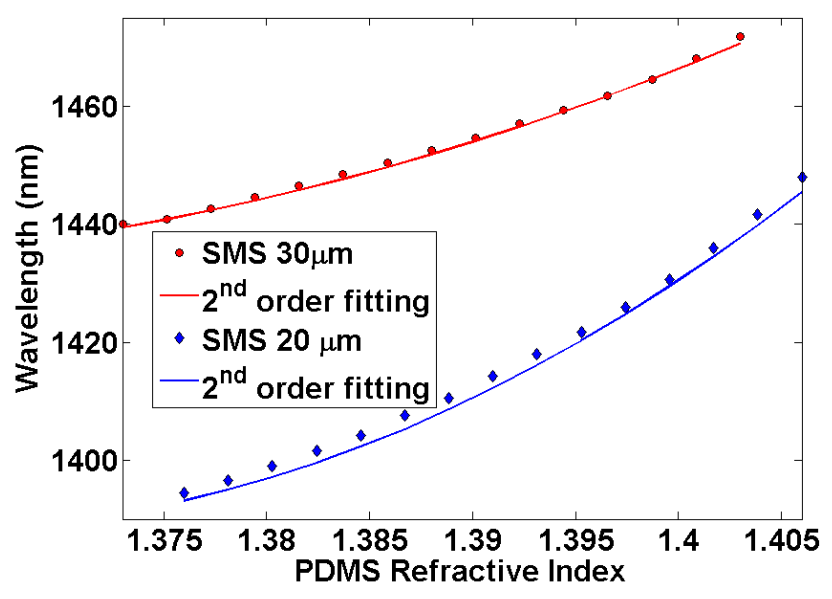

Fig. 13. Theoretical wavelength shifts for the different devices studied

From Fig. 13 above, it can be seen that the sensitivity was higher for the device with a smaller diameter, as reported previously in the literature [8], and the enhancement factor was approximately 1.5 , which is close to the factor of 1.75 reported in the bibliography. The experimental and theoretical changes in the sensitivity can now be compared. From the experimental data, we can extract the sensitivity of device D3 $(20 \mu \mathrm{m}, 6 \mathrm{~mm}$ and RTV615 PDMS): its wavelength shift was $12 \mathrm{~nm}$, while for the same pressure variation, D2 showed a wavelength shift of $3.5 \mathrm{~nm}$, i.e. lower by a factor of about four. In addition, since the sensitivity of these devices changes in a linear way with the decimal logarithm of the pressure over a certain range, we can calculate the experimental sensitivity as $5.57 \mathrm{~nm} / \log (\mathrm{mbar})$ for device D3 and $2.17 \mathrm{~nm} / \log (\mathrm{mbar})$ for device D2, if only the linear response is considered, as shown in Fig. 14.

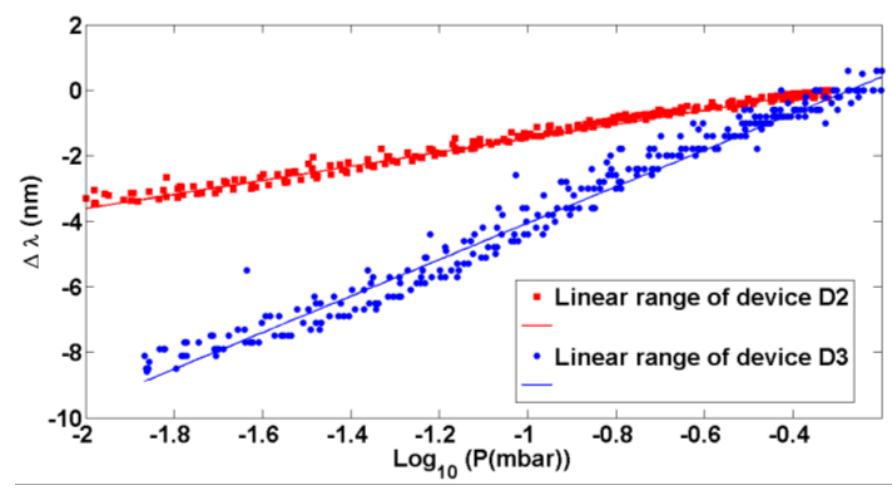

Fig. 14. Experimental wavelength shift, limited to the range in which the sensors show a linear response

We can observe that the enhancement factor is 2.567 , a value that is greater than expected due to the diameter reduction. Furthermore, the range in which the sensor is sensitive and the response is linear is slightly different when RTV615 is used instead of Sylgard 184; the linearity is also greater for device D2, with an R-square coefficient of 0.9859 for device D2 and 0.9764 for device D3. We can therefore conclude that the elastic properties of the material have an influence on the behavior of the sensor. Hence, from a comparison between the expected and 
obtained sensitivities, we conclude that the material used also has an effect on the improvement in the sensitivity.

\section{CONCLUSION}

A novel optical fiber vacuum sensor was developed using an etched SMS modal interferometer. This sensor was different from other optical pressure sensors, which are generally based on membranes and Fabry-Pèrot interferometers or on thermal conductivity. This structure was chosen for its simplicity of fabrication and for its sensitivity to changes in strain and refractive index. It is also feasible to work with modal interference using other types of fibers that can improve the transmitted optical spectrum.

The proposed sensor shows good behavior in the $1 \times 10^{-3}$ to 1 mbar range, with a dynamic range of $12 \mathrm{~nm}$. The highest sensitivity, which occurs in the range 0.01 to 0.3 mbar, is 35 $\mathrm{nm} / \mathrm{mbar}$. This sensitivity was obtained by the device with the minimum diameter studied here of $20 \mu \mathrm{m}$, and with the use of toluene as a solvent for the RTV-615 PDMS. We conclude that the elastic properties of the PDMS used here influence the sensitivity of the device, the range of measurement and the response time. The use of toluene in higher proportions or coating the PDMS by other methods may improve the thickness control and the morphological properties of the coating. A more exhaustive study of the differences between the degree of crosslinking in PDMS and the different co-polymers will be necessary in future.

In addition, there are optical structures with greater sensitivities that might offer the possibility of working in other pressure ranges, such as lossy mode resonances, which can extend the range of pressures that can be measured, improve the robustness of the fabricated devices and increase the sensitivity in this range.

\section{ACKNOWLEDGMENTS}

Joaquin Ascorbe would like to thank Ministerio de Ciencia e Innovación for Torres-Quevedo research grant PTQ2018010221.

\section{REFERENCES}

[1] [1] P. Roriz, O. Frazão, A. B. Lobo-Ribeiro, J. L. Santos, and J. A. Simões, "Review of fiber-optic pressure sensors for biomedical and biomechanical applications," J. Biomed. Opt., vol. 18, no 5, p. 050903. 2013.

[2] W. J. Alvesteffer, D. C. Jacobs, and D. H. Baker, "Miniaturized thin film thermal vacuum sensor," J. Vac. Sci. Technol. A, vol. 13, no. 6, pp. 2980 2985,1995

[3] F. Urban, J. Kadlec, R. Vlach, and R. Kuchta, "Design of a pressure sensor based on optical fiber Bragg grating lateral deformation," Sensors, vol. 10, no. 12, pp. 11212-11225, 2010

[4] K. Totsu, Y. Haga and M. Esashi, "Vacuum Sealed Ultra Miniature FiberOptic Pressure Sensor Using White Light Interferometry," in 12th International Conference on Solid-State Sensors, Actuators and
Microsystems, pp. 931-934, 2003.

[5] X. Wang, J. Xu, Y. Zhu, K. L. Cooper, and A. Wang, "All-fused-silica miniature optical fiber tip pressure sensor," vol. 31, no. 7, pp. 885-887, 2006.

[6] B. McMillen, C. Jewart, M. Buric, K. P. Chen, Y. Lin, and W. Xu, "Fiber Bragg grating vacuum sensors," Appl. Phys. Lett., vol. 87, no. 23, pp. 1$3,2005$.

[7] O. Fuentes et al., "Increasing the Sensitivity of an Optic Level Sensor with a Wavelength and Phase Sensitive Single-Mode Multimode Single-Mode Fiber Structure,” IEEE Sens. J., vol. 17, no 17, p. 5515-5522. 2017.

[8] Y. Cardona-Maya, I. Del Villar, A. B. Socorro, J. M. Corres, I. R. Matias, and J. F. Botero-Cadavid, "Wavelength and Phase Detection Based SMS Fiber Sensors Optimized with Etching and Nanodeposition," J. Light. Technol., vol. 35, no 17, p. 3743-3749. 2017.

[9] R. M. André, C. R. Biazoli, S. O. Silva, M. B. Marques, C. M. B. Cordeiro, and O. Frazão, "Multimode interference in tapered single modemultimode-single mode fiber structures for strain sensing applications," in Proc. OFS2012 22nd International Conference on Optical Fiber Sensors; 2012, 84213B

[10] A. B. Socorro et al., "Fiber-Optic Immunosensor Based on an Etched SMS Structure," IEEE J. Sel. Top. Quantum Electron., vol. 23, no 2, p. 314-321, 2017

[11] S. Silva et al., "Ultrahigh-sensitivity temperature fiber sensor based on multimode interference.," Appl. Opt., vol. 51, no 16, p. 3236-3242, 2012.

[12] A. M. Hatta, Y. Semenova, Q. Wu, and G. Farrell, "Strain sensor based on a pair of single-mode-multimode-single-mode fiber structures in a ratiometric power measurement scheme," Appl. Opt., vol. 49, no 3, p. 536-541, 2010.

[13] Q. Wu, Y. Semenova, P. Wang, A. M. Hatta, and G. Farrell, "Experimental demonstration of a simple displacement sensor based on a bent single-mode-multimode-single-mode fiber structure," Meas. Sci. Technol., vol. 22, no 2, p. 025203, 2011.

[14] A. Mehta, W. Mohammed, and E. G. Johnson, "Multimode interferencebased fiber-optic displacement sensor," IEEE Photonics Technol. Lett., vol. 15, no 8, p. 1129-1131. 2003.

[15] A. W. Snyder and J. D. Love, Optical Waveguide Theory. Springer US, 1984.

[16] J. Zheng, L. Pei, T. Ning, J. Li, C. Zhang, S. Ma, and Z. Ruan, "Matching optimization for SFS-structured interferometers with step-index fibers," Opt. Express, vol. 26, no. 7, pp. 9182-9193, 2018.

[17] S. Hyun, C. Cohen, and C. Hui, "Mechanical and swelling properties of PDMS interpenetrating polymer networks," Polymer, vol. 47, pp. 62266235, 2006.

[18] M. Mike, Y. Huang, K. Choi, and C. H. Hidrovo, "The improved resistance of PDMS to pressure-induced deformation and chemical solvent swelling for microfluidic devices," Microelectronic engineering, vol. 124, pp. 66-75, 2014.

[19] SYLGARDTM 184, "Technical Data Sheet, The Dow Corning Company."

[20] S. Silva, E. G. P. Pachon, M. A. R. Franco, et al., "Ultrahigh-sensitivity temperature fiber sensor based on multimode interference," Appl. Opt., vol. 51, no. 16, pp. 3236-3242, 2012.

[21] I. Del Villar, A. B. Socorro, J. M. Corres, F. J. Arregui and I. R. Matias, "Optimization of Sensors Based on Multimode Interference in SingleMode - Multimode - Single-Mode Structure," J. Light. Technol., vol. 31, no. 22 , pp. 3460-3468, 2013.

[22] I. Del Villar, A. B. Socorro, J. M. Corres, F. J. Arregui, and I. R. Matias, "Refractometric sensors based on multimode interference in a thin-film coated single-mode-multimode-single-mode structure with reflection configuration,” Appl. Opt., vol. 53, no. 18, pp. 3913-3919, Jun. 2014. 